\title{
Rearrangement of the Chromatin Organizer Special AT-rich Binding Protein 1 Gene, SATB1, Resulting from a $t(3 ; 5)(p 24 ; q 14)$ Chromosomal Translocation in Acute Myeloid Leukemia
}

\author{
SYNNE TORKILDSEN ${ }^{1,2,3}$, MARTA BRUNETTI ${ }^{1,2}$, LUDMILA GORUNOVA $^{1,2}$, \\ SIGNE SPETALEN $^{4}$, KLAUS BEISKE ${ }^{4}$, SVERRE HEIM $^{1,2,5}$ and IOANNIS PANAGOPOULOS ${ }^{1,2}$ \\ ${ }^{1}$ Section for Cancer Cytogenetics, Institute for Cancer Genetics and Informatics, \\ The Norwegian Radium Hospital, Oslo University Hospital, Oslo, Norway; \\ ${ }^{2}$ Centre for Cancer Biomedicine, Faculty of Medicine, University of Oslo, Oslo, Norway; \\ Departments of ${ }^{3}$ Hematology and ${ }^{4}$ Pathology, Oslo University Hospital, Oslo, Norway; \\ ${ }^{5}$ Faculty of Medicine, University of Oslo, Oslo, Norway
}

\begin{abstract}
Background/Aim: New chromosomal aberrations continue to be reported in acute myeloid leukemias (AML). The addition of more cases with the same genetic characteristics would establish an acquired aberration as a recurrent change, help determine its prognostic significance, and can provide insight into the mechanisms of leukemogenesis in patients with these rare abnormalities. Case Report: RNA-sequencing was performed on a patient with $A M L$ with the bone marrow karyotype $46, X Y, t(3 ; 5)(p 24 ; q 14)[5] / 46, X Y[10]$. The translocation resulted in fusion of the SATB homeobox 1 gene (SATB1) (3p24) with an expression sequence tag with accession number BG503445 (5q14). The SATB1-BG503445 transcript may code for a SATBl protein that would lack the $C$ terminal DNA-binding homeodomain. Conclusion: The present study is the first to demonstrate rearrangement and disruption of SATBI in AML. Rearrangements of chromosome band $3 p 24$ were reported in 24 additional AMLs but not in known leukemia-specific chromosomal abnormalities. Further studies are needed to determine whether SATB1-BG503445 or other aberrations of SATB1 are recurrent in $A M L$.
\end{abstract}

Correspondence to: Ioannis Panagopoulos, Section for Cancer Cytogenetics, Institute for Cancer Genetics and Informatics, The Norwegian Radium Hospital, Oslo University Hospital, P.O.Box 4953 Nydalen, NO-0424 Oslo, Norway. Tel: +47 22782363, e-mail: ioannis.panagopoulos@rr-research.no

Key Words: Acute myeloid leukemia, chromosome translocation, RNA-sequencing, SATB1 gene, truncation.
Over the past two decades, acute myeloid leukemia (AML) has been transformed from a disease defined, classed, and staged based on cytological and other hematological characteristics alone to a disease classified largely based on genetic, genomic, and molecular characteristics $(1,2)$. Cytogenetic analysis of bone marrow cells has become an integral part of the clinical management of patients with hematological malignancies, not least AML, yielding diagnostic as well as prognostic information (http://www.uptodate.com/contents/cytogenetics-inacute-myeloid-leukemia). Sometimes this type of information is decisive in choosing the optimal treatment for patients with AML as illustrated most pertinently by the finding at diagnosis of the chromosomal translocation $\mathrm{t}(15 ; 17)(\mathrm{q} 22 ; \mathrm{q} 21)$, the hallmark of acute promyelocytic leukemia, an AML subtype treated and monitored differently from other subtypes (3).

The detection of acquired chromosomal abnormalities in leukemia cells, in particular translocations and inversions, has helped identify genes located in the breakpoints that, when rearranged or otherwise deregulated, launch or contribute to the leukemogenic process (4). Numerous pathogenetic or genetic subgroups have thus been identified (4). Nevertheless, new recurrent or unique chromosomal aberrations (mainly balanced translocations) corresponding to smaller cytogenetic subgroups continue to be reported in AML and myelodysplastic syndromes (MDS) (5). The addition of more cases with the same genetic characteristics will establish the acquired aberration in question as a recurrent change, but may also help determine the prognostic significance of the aberration, as well as provide insights into the mechanisms of leukemogenesis in patients with these rare abnormalities $(5)$.

We report here a $\mathrm{t}(3 ; 5)(\mathrm{p} 24 ; \mathrm{q} 14)$ in a case of AML which resulted in the fusion of the SATB homeobox 1 gene 
(SATB1) (3p24) with an expression sequence tag with accession number $B G 503445$ from $5 \mathrm{q} 14$, leading to the generation of a putative SATB1 truncated protein.

Ethics statement. The study was approved by the Regional Committee for Medical and Health Research Ethics, SouthEast Norway (REK Sør- øst, S-07474a). Written informed consent was obtained from the patient prior to his death. The Ethics Committee's approval included a review of the consent procedure. All patient information was anonymized.

\section{Case Report}

A 65-year-old man was diagnosed with MDS, classified as refractory cytopenia with multilineage dysplasia, in May 2013. No excess of blasts were described in the bone marrow smear at diagnosis, but a trisomy 8 chromosomal aberration was detected (see below). The patient received blood transfusions every other week until progression to AML occurred 15 months later. Examination of a bone marrow aspirate then showed normal hematopoiesis to be replaced by $50 \%$ blasts. Immunophenotypic analysis confirmed the myeloid origin of the blasts that were positive for cluster of differentiations (CD) CD34, CD117, and CD56. The CD34 count was only $7 \%$, probably because the aspirate was heavily admixed with blood. Molecular genetic analysis was negative for fms related tyrosine kinase 3 (FLT3), nucleophosmin (NPMI), and CCAAT/enhancer binding protein alpha (CEBPA) mutations, and none of the most common AML-specific fusion transcripts were detected using the HemaVision 28Q RT-qPCR -KIT (DNA Diagnostic, Risskov, Denmark).

The patient received induction therapy for AML with $90 \mathrm{mg} / \mathrm{m}^{2}$ daunorubicin days $1-3$ and $200 \mathrm{mg} / \mathrm{m}^{2}$ cytarabine days 1-7. Although morphological remission was obtained, a persistent and significant aplasia remained even 4 months after induction treatment. It was therefore decided to stop further cytostatic treatment. Two months later, the patient was admitted to a local hospital due to dizziness, difficulties in finding words, memory loss, and headache. Magnetic resonance imaging showed a meningeal affection over most of the left hemisphere with mass effect, perceived as a manifestation of AML relapse, confirmed by examination of a new bone marrow aspirate. The patient was therefore treated with high-dose steroids and palliative radiotherapy (18 Gy), as well as hydroxyurea because of a stark increase in leukocyte and lactate dehydrogenase level. Despite this, the patient died 10 days after the beginning of radiation therapy.

G-Banding analysis. Bone marrow cells were cytogenetically investigated by standard methods. Chromosomal preparations were made from metaphase cells of a 24-h culture, G-banded using Leishman stain, and karyotyped according to International System for Human Cytogenetic Nomenclature 2013 guidelines (6).

Fluorescence in situ hybridization (FISH). As part of our standard cytogenetic diagnosis, initial interphase FISH analyses of bone marrow cells were performed using the Cytocell multiprobe AML/MDS panel (Cytocell, Cambridge, UK) looking for $5 / \operatorname{del}(5 q), 7 / \operatorname{del}(7 q)$, del(20q), deletion of 17p13 (TP53), lysine methyltransferase 2A (KMT2A also known as $M L L$ ) rearrangements, promyelocytic leukemiaretinoic acid receptor alpha $(P M L-R A R A)$ fusion created by $\mathrm{t}(15 ; 17)(\mathrm{q} 22 ; \mathrm{q} 21)$, runt related transcription factor 1 RUNX1 translocation partner 1 (RUNX1-RUNX1T1) created by $\mathrm{t}(8 ; 21)(\mathrm{q} 22 ; \mathrm{q} 22)$, and core binding factor beta- myosin heavy chain 11 (CBFB-MYH11) generated by the inversion $\operatorname{inv}(16)(\mathrm{p} 13 \mathrm{q} 22)$. Fluorescent signals were captured and analyzed using the CytoVision system (Leica Biosystems, Newcastle-upon-Tyne, UK).

RNA-sequencing. Three micrograms of total RNA extracted from the patient's bone marrow at the time of progression to AML were sent for high-throughput paired-end RNA sequencing at the Norwegian Sequencing Centre at Ullevål Hospital (http://www.sequencing.uio.no/). Detailed information about these analyses was given elsewhere (7). A total of 123 million reads were obtained. FASTQC software was used for quality control of the raw sequence data (http://www.bioinformatics.babraham.ac.uk/projects/fastqc/).

TopHat-Fusion software was used for the discovery of fusion transcripts $(8,9)$.

Reverse transcription polymerase chain reaction (RT-PCR) analyses. For RT-PCR, $1 \mu \mathrm{g}$ of total RNA was reversetranscribed in a 20- $\mu$ l reaction volume using iScript Advanced cDNA Synthesis Kit for RT-qPCR according to the manufacturer's instructions (Bio-Rad Laboratories, Oslo, Norway). The $25 \mu \mathrm{l}$ PCR volume contained $12.5 \mu \mathrm{l}$ Premix Ex Taq $^{\mathrm{TM}}$ DNA Polymerase Hot Start Version (Takara Bio Europe/SAS, Saint-Germain-en-Laye, France), $1 \mu \mathrm{l}$ of cDNA, and $0.4 \mu \mathrm{M}$ of each of the forward and reverse primers. The quality of the cDNA synthesis was assessed by amplification of a cDNA fragment of the ABL protooncogene 1, non-receptor tyrosine kinase $(A B L 1)$ gene using the primers ABL1-91F1 (5'-CAG CGG CCA GTA GCA TCT GAC TTT G-3') and ABL1-404R1 (5' CTC AGC AGA TAC TCA GCG GCA TTG C-3') (7). Two primer combinations were used: The forward primer SATB1-3246F1 (5'-AGG AAA TGA AGC GTG CTA AAG TGT-3') together with the reverse BG503445-359R1 (5'-CCT CTG AGG TTT ATG ACT CCA TGC-3') and the forward SATB1-3147F1 (5'-CTC CCC AGG TGA AAA CAG CTA CTA-3') with the 
Table I. Fusions between chromosomal bands $3 p 24$ and $5 q 14$ which were found with the TopHat-Fusion program. The sequences corresponding to the SATB1-BG503445 fusion transcript are in bold.

\begin{tabular}{lccc}
\hline p24-Fusion point & 5q14-Fusion point & Fifty bases on the left side of the fusion (3p24) & Fifty bases on the right side of the fusion (5q14) \\
\hline 18419661 & 88600566 & CTAAAGTGTCTCAAGCACTGTTTGCA & GAATTCTACTAGTGCATGTGGAGAGA \\
& & AAGGTTGCAGCAACCAAAAGCCAG & AACATGGCTGCTGCATGGAGTCAT \\
22423821 & 77940164 & ACGAAAAGGATATTGCTGCATATCGAG & AAAAAAGTGAGTTGTCAAGGCTGAAAA \\
& CTAAAGGAAAGCCTGATGCAGCA & AAGCAAGAAAAAGAAGGAAGAGG \\
22423828 & 77940156 & GGATATTGCTGCATATCGAGCTAAAGGA & GAGTTGTCAAGGCTGAAAAAAGCAAGA \\
& AAGCCTGATGCAGCAAAAAAGG & AAAAGAAGGAAGAGGAGGAAGAT \\
\hline
\end{tabular}

reverse BG503445-422R1 (5'-TCT TTC TTG CCA AAC TTG CCA TA-3'). The primers SATB1-3246F1 and SATB13147F1 correspond to positions 3246-3269 and 3147-3170 in the $S A T B 1$ reference sequence with accession number NM_002971.3. The reverse BG503445-359R1 and BG503445-422R1 primers correspond to positions 382-359 and 444-422 in the sequence with accession number BG503445.1. The PCRs were run on a C-1000 Thermal cycler (Bio-Rad Laboratories, Oslo, Norway) with an initial denaturation at $94^{\circ} \mathrm{C}$ for $30 \mathrm{~s}$, followed by 35 cycles of $7 \mathrm{~s}$ at $98^{\circ} \mathrm{C}, 30 \mathrm{~s}$ at $55^{\circ} \mathrm{C}, 30 \mathrm{~s}$ at $72^{\circ} \mathrm{C}$, and a final extension for 5 min at $68^{\circ} \mathrm{C}$. Three microliters of the PCR products were stained with GelRed (Biotium, Hayward, CA, USA), analyzed by electrophoresis through $1.0 \%$ agarose gel, and photographed. The remaining $22 \mu \mathrm{l}$ PCR products were purified using the MinElute PCR purification kit (Qiagen Nordic, Oslo, Norway) and direct sequenced using the dideoxy procedure with ABI Prism BigDye terminator v1.1 cycle sequencing kit (ThermoFisher Scientific, Oslo, Norway) on the Applied Biosystems Model 3500 Genetic Analyzer sequencing system (ThermoFisher Scientific, Oslo, Norway). BLAST software (http://blast.ncbi.nlm.nih.gov/ Blast.cgi) was used for computer analysis of sequence data.

\section{Results}

Karyotyping and FISH. G-Banding analysis of bone marrow cells at diagnosis (myelodysplastic syndrome-refractory cytopenia with multilineage dysplasia) yielded the karyotype $47, \mathrm{X},+8[2] / 46, \mathrm{XY}[15]$. However, 15 months later when the disease had progressed to AML, the G-banding analysis of bone marrow cells yielded a different karyotype: 46,XY,t(3;5)(p24;q14)[5]/46,XY[10] (Figure 1A) with not one cell showing trisomy 8 . FISH analysis showed no 5/del(5q), -7/del(7q), del(20q), deletion of TP53, MLL rearrangements, or $P M L-R A R A, R U N X 1-R U N X 1 T 1$, or $C B F B-M Y H 11$ fusion. In particular, the RUNX1T1 (8q22) probe showed only two green signals in all examined interphase nuclei confirming absence of trisomy 8 at this point in disease development.
Analysis of RNA-sequencing. Using the TopHat-Fusion on the raw sequencing data obtained by Norwegian Sequencing Centre, three fusions were found between chromosome bands 3 p24 and 5q14 (Table I). Whereas two of them recombined non-genic and seemingly uninteresting sequences, the third was a fusion between $S A T B 1$ (from $3 \mathrm{p} 24)$ and the sequence with accession number BG503445, which is an expressed sequence tag that has been spliced, mapped within the myocyte enhancer factor 2C (MEF2C) antisense RNA 1 locus (http://www.ncbi.nlm.nih.gov/ gene/?term=MEF2C-AS1), and found to be expressed in embryonal carcinoma (https://www.ncbi.nlm.nih.gov/ nucest/BG503445).

Molecular confirmation of the SATB1-BG503445 fusion. PCR with the primers ABL1-91F1 and ABL1-404R1 amplified cDNA fragments of $A B L 1$, indicating that the synthesized cDNAs were of good quality (Figure 1B).

PCR with the primer sets SATB1-3246F1/BG503445359R1 and SATB1-3147F1/ BG503445-422R1 amplified a $120 \mathrm{bp}$ and a $280 \mathrm{bp}$ cDNA fragment, respectively (Figure 1B). Direct sequencing of the amplified fragments verified the presence of the SATB1-BG503445 transcript, with the fusion point identical to that found with TopHat (Figure 1C).

\section{Discussion}

We present a case of AML in which the leukemia cells had a novel $\mathrm{t}(3 ; 5)(\mathrm{p} 24 ; \mathrm{q} 14)$ chromosomal translocation as the sole cytogenetic abnormality. The molecular analysis of the translocation showed that it resulted in fusion of the SATB1 gene with the expressed sequence tag BG503445.

The $S A T B 1$ gene is expressed predominantly in the thymus and encodes a matrix protein which binds nuclear matrix and scaffold-associating DNAs through a unique nuclear architecture (10). It is required for development of thymocytes and activation of helper T cells 2 (Th2) cells. Without the SATB1 protein, thymocyte development is blocked at the $\mathrm{CD} 4^{+} \mathrm{CD} 8^{+}$stage and Th2 cells cannot become activated and produce cytokines (10). The SATB1 protein was 

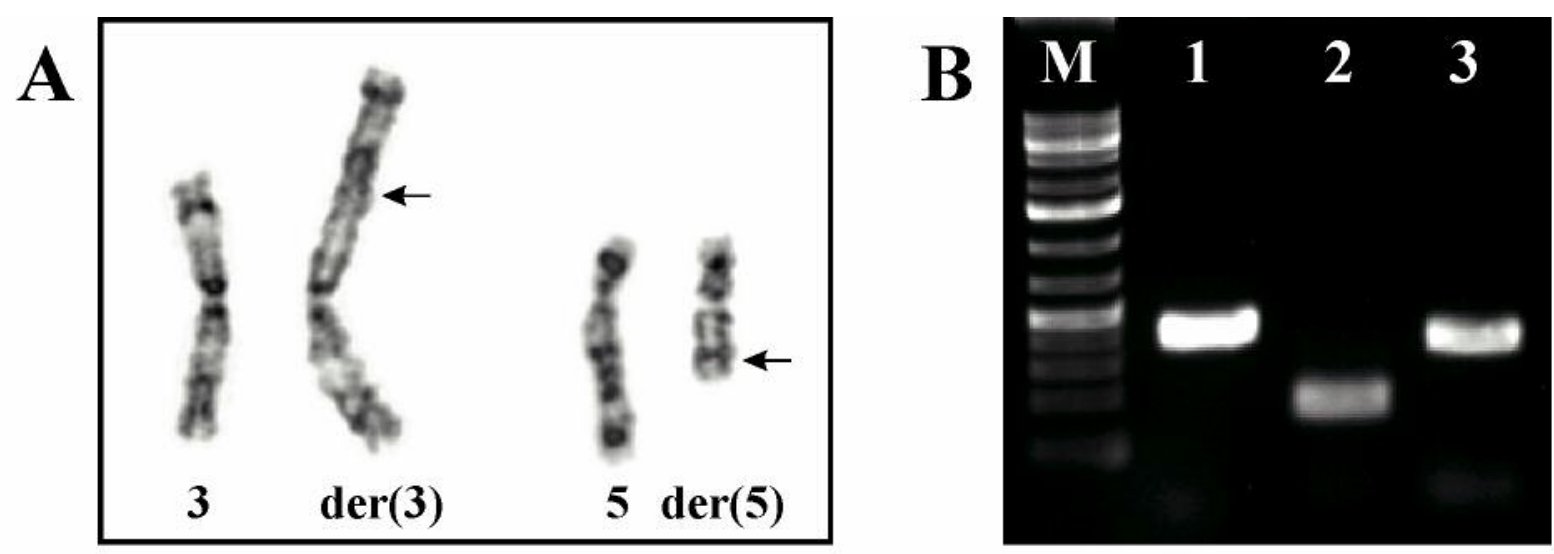

\section{SATB1 exon $9 \downarrow B G 503445$ nt 321}

T T G C G CA C CA A A C CA G A T T TACTA T T TA T T G

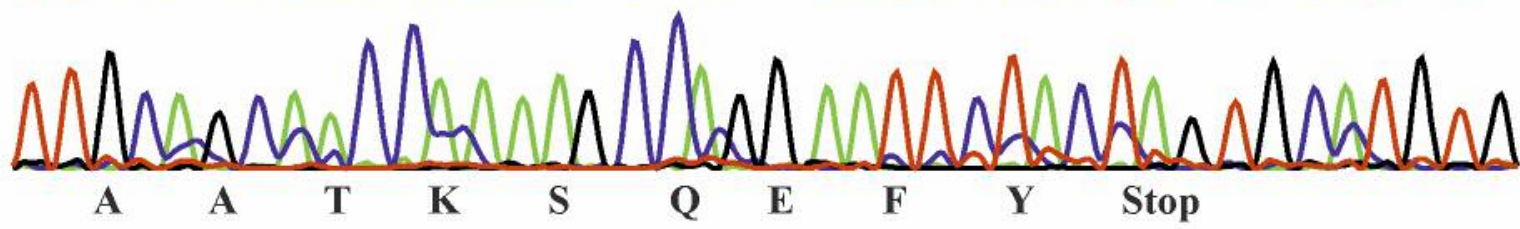

D

NP_002962.1:0..763 DNA-binding protein SATB1 isoform 1

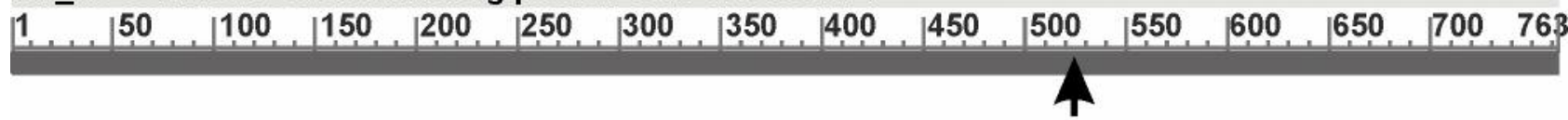

\section{SATB1_N}

PDZ-like

dimerization

Nuclear

localization

signal

\begin{abstract}
domain
\end{abstract}

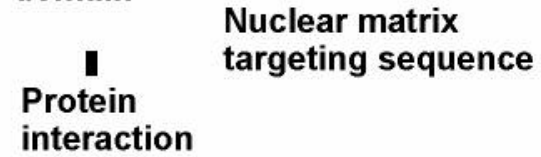

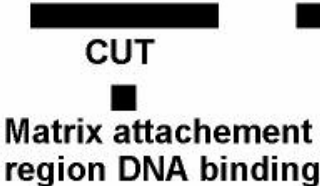

CUT

Homeodomain

Figure 1. Cytogenetic and molecular genetic analysis of bone marrow cells from the patient. A: Partial karyotype showing the der(3)t(3;5)(p24;q14) and der(5)t(3;5)(p24;q14) together with the corresponding normal chromosome homologs. Breakpoint positions are indicated by arrows. B: Gel electrophoresis showing the amplified cDNA fragments. Lane 1, amplification of an ABL proto-oncogene 1, non-receptor tyrosine kinase (ABL1) cDNA fragment using the primers ABL1-91F1 and ABL1-404R1. Lanes 2 and 3, amplifications of cDNA fragments using the primer combinations SATB1-3246F1/BG503445-359R1 and SATB1-3147F1/ BG503445-422R1, respectively. M, $1 \mathrm{~kb}$ DNA ladder (GeneRuler, ThermoFisher). C: Partial sequence chromatogram of the amplified cDNA fragment showing the junction point of the SATB homeobox 1 (SATB1) gene with the sequence with accession number BG503445. D: Illustration of the SATB1 protein and its functional domains. Arrow shows the breakpoint. PDZ: PSD95/Dlg/ZO1 (PDZ)-like dimerization domain; CUT: CUT DNA binding motif. 
also shown to play an important role in hematopoietic stem cells self-renewal, in lymphoid lineage specification, in epidermal differentiation, and brain function (10-12).

SATB1 folds chromatin into loops and recruits chromatin remodeling and modifying enzymes to these DNA loops. Depending on the genomic location, SATB1 attracts histone deacetylases and other histone modifying enzymes to the site and a change in histone marks occurs that may either activate or repress transcription (11). SATB1 controls a plethora of genes many of which [erb-b2 receptor tyrosine kinase 2 (ERRB2), ABL1, matrix metallopeptidase 2 (MMP2), Ecadherin $(C D H 1)$, vascular endothelial growth factor $\mathrm{B}$ $(V E G F B)$, transforming growth factor beta $1(T G F B 1)$, and others] are involved in carcinogenesis (10-12). Thus, expression of $S A T B 1$ is associated with many types of cancer, including colorectal, breast, pancreatic, nasopharyngeal, bladder, prostate, lung, ovarian, liver, glioma, lymphoma, and kidney tumors (11). In most cancer types, $S A T B 1$ expression is associated with progression and poor prognosis [(11) and references therein].

SATB 1 has been linked to AML [(10) and references therein]. Disruption of the distal enhancer of the Spi-1 protooncogene, SPI1, gene (also known as PU.1), coding for an E26 transformation-specific (ETS)-domain transcription factor, reduces its expression leading to AML in mice. A single nucleotide polymorphism which is frequently associated with human AML, was identified within the distal enhancer of the SPII gene and specifically inhibits SATB1 binding, causing reduced SPII expression in myeloid progenitor cells [(10) and references therein].

The present study is the first to demonstrate rearrangement and disruption of $S A T B 1$ in hematological malignancies stemming from the chromosomal translocation $\mathrm{t}(3 ; 5)(\mathrm{p} 24 ; \mathrm{q} 14)$.

Molecular analysis of the translocation showed fusion of the $S A T B 1$ gene with a sequence with accession number $B G 503445$ from $5 \mathrm{q} 14$ resulting in a putative SATB1 truncated protein (Figure 1). This protein would contain the nuclear localization signal domain, the $\mathrm{N}$-terminal domain of SATB1 which is involved in various interactions with chromatin proteins, the PSD95/Dlg/ZO-1 (PDZ)-like dimerization domain, the nuclear matrix targeting sequence, and the first CUT DNA binding motif (Figure 1D). It would lack the C-terminal homeodomain DNA binding domain which binds to DNA as a monomer or homo- or heterodimer playing a part in the transcriptional regulation of key eukaryotic developmental processes (13). The precise role of SATB1 truncated protein in the development of leukemia cannot be predicted without functional studies. However, anomaly in transcription regulation can be assumed. Alternatively, loss of a functional $S A T B 1$ allele might be the important factor in leukemogenesis.

Chromosomal aberrations resulting in gene truncation have been repeatedly described for the RUNXI and ETS variant 6 (ETV6) genes $(14,15)$. The translocation generates a premature stop codon in the open reading frames leading to expression of C-terminal truncated forms of the RUNX1 or ETV6 proteins $(14,15)$. Truncated RUNX1 proteins interfere with normal RUNX1 protein (16-18). Truncated forms of ETV6 were shown to have a dominant-negative effect on normal ETV6 function and disrupt both primitive and definitive hematopoiesis in the zebrafish model (19).

In the Mitelman Database of Chromosome Aberrations and Gene Fusions in Cancer (http://cgap.nci.nih.gov/ Chromosomes/Mitelman; at May 4, 2016), 24 cases of AML can be found with breakpoints in chromosome band $3 \mathrm{p} 24$ where SATB1 maps. SATB1 may or may not be pathogenetically involved in these leukemias. The same goes for other cases with breakpoints mapped near $3 \mathrm{p} 24$, for example, for two myeloid leukemias with $\mathrm{t}(3 ; 5)(\mathrm{p} 21 ; \mathrm{q} 13-14)$ $(20,21)$; the seeming cytogenetic variability is not so large that it might not be due to interpretation difficulties. Greater awareness of the role of SATBI in leukemic contexts certainly seems warranted.

\section{Competing Interests}

The Authors declare that they have no competing interests.

\section{Acknowledgements}

This work was supported by grants from the Norwegian Radium Hospital Foundation.

\section{References}

1 Papaemmanuil E, Gerstung M, Bullinger L, Gaidzik VI, Paschka P, Roberts ND, Potter NE, Heuser M, Thol F, Bolli N, Gundem G, Van Loo P, Martincorena I, Ganly P, Mudie L, McLaren S, O’Meara S, Raine K, Jones DR, Teague JW, Butler AP, Greaves MF, Ganser A, Dohner K, Schlenk RF, Dohner H and Campbell PJ: Genomic classification and prognosis in acute myeloid leukemia. N Engl J Med 374: 2209-2221, 2016.

2 Vardiman JW, Thiele J, Arber DA, Brunning RD, Borowitz MJ, Porwit A, Harris NL, Le Beau MM, Hellstrom-Lindberg E, Tefferi A and Bloomfield CD: The 2008 revision of the World Health Organization (WHO) Classification of myeloid Neoplasms and Acute Leukemia: rationale and important changes. Blood 114: 937-951, 2009.

3 Cull EH and Altman JK: Contemporary treatment of APL. Curr Hematol Malig Rep 9: 193-201, 2014.

4 Heim S and Mitelman F: Cancer Cytogenetics: Chromosomal and Molecular Genetic Abberations of Tumor Cells: WileyBlackwell, Chichester, West Sussex, UK, 2015.

5 Walker A, Mrozek K, Kohlschmidt J, Rao KW, Pettenati MJ, Sterling LJ, Marcucci G, Carroll AJ, Bloomfield CD and Alliance for Clinical Trials in O: New recurrent balanced translocations in acute myeloid leukemia and myelodysplastic syndromes: cancer and leukemia group B 8461. Genes Chromosomes Cancer 52: 385-401, 2013. 
6 Schaffer LG, McGowan-Jordan J and Schmid M: ISCN 2013 An International System for Human Cytogenetic Nomenclature. Basel: Karger, 2013.

7 Torkildsen S, Gorunova L, Beiske K, Tjonnfjord GE, Heim S and Panagopoulos I: Novel ZEB2-BCL11B fusion gene identified by RNA-sequencing in acute myeloid leukemia with $\mathrm{t}(2 ; 14)(\mathrm{q} 22 ; \mathrm{q} 32)$. PLoS One 10: e0132736, 2015.

8 Kim D, Pertea G, Trapnell C, Pimentel H, Kelley R and Salzberg SL: TopHat2: accurate alignment of transcriptomes in the presence of insertions, deletions and gene fusions. Genome Biol 14: R36, 2013.

9 Kim D and Salzberg SL: TopHat-Fusion: an algorithm for discovery of novel fusion transcripts. Genome Biol 12: R72, 2011.

10 Kohwi-Shigematsu T, Poterlowicz K, Ordinario E, Han HJ, Botchkarev VA and Kohwi Y: Genome-organizing function of SATB1 in tumor progression. Semin Cancer Biol 23: 72-79, 2013.

11 Brocato $\mathrm{J}$ and Costa M: SATB1 and 2 in colorectal cancer Carcinogenesis 36: 186-191, 2015.

12 Satoh Y, Yokota T, Sudo T, Kondo M, Lai A, Kincade PW, Kouro T, Iida R, Kokame K, Miyata T, Habuchi Y, Matsui K, Tanaka H, Matsumura I, Oritani K, Kohwi-Shigematsu T and Kanakura Y: The SATB1 protein directs hematopoietic stem cell differentiation toward lymphoid lineages. Immunity 38: 11051115, 2013.

13 Bürglin TR and Affolter M: Homeodomain proteins: an update. Chromosoma 125: 497-521, 2016.

14 De Braekeleer E, Douet-Guilbert N, Morel F, Le Bris MJ, Basinko A and De Braekeleer M: ETV6 fusion genes in hematological malignancies: a review. Leuk Res 36: 945-961, 2012.

15 De Braekeleer E, Douet-Guilbert N, Morel F, Le Bris MJ, Ferec $\mathrm{C}$ and De Braekeleer M: RUNX1 translocations and fusion genes in malignant hemopathies. Future Oncol 7: 77-91, 2011.

16 Hromas R, Busse T, Carroll A, Mack D, Shopnick R, Zhang DE, Nakshatri $\mathrm{H}$ and Richkind $\mathrm{K}$ : Fusion $A M L 1$ transcript in a radiation-associated leukemia results in a truncated inhibitory AML1 protein. Blood 97: 2168-2170, 2001.
17 Ramsey H, Zhang DE, Richkind K, Burcoglu-O'Ral A and Hromas R: Fusion of $A M L 1 / R U N X 1$ to copine VIII, a novel member of the copine family, in an aggressive acute myelogenous leukemia with $\mathrm{t}(12 ; 21)$ translocation. Leukemia 17: 1665-1666, 2003.

18 Rodriguez-Perales S, Torres-Ruiz R, Suela J, Acquadro F, Martin MC, Yebra E, Ramirez JC, Alvarez S and Cigudosa JC: Truncated RUNX1 protein generated by a novel $t(1 ; 21)(\mathrm{p} 32 ; \mathrm{q} 22)$ chromosomal translocation impairs the proliferation and differentiation of human hematopoietic progenitors. Oncogene 35: 125-134, 2016.

19 Rasighaemi P, Liongue C, Onnebo SM and Ward AC: Functional analysis of truncated forms of ETV6. Br J Haematol 171: 658662, 2015.

20 Mallo M, Arenillas L, Espinet B, Salido M, Hernandez JM, Lumbreras E, del Rey M, Arranz E, Ramiro S, Font P, Gonzalez O, Renedo M, Cervera J, Such E, Sanz GF, Luno E, Sanzo C, Gonzalez M, Calasanz MJ, Mayans J, Garcia-Ballesteros C, Amigo V, Collado R, Oliver I, Carbonell F, Bureo E, Insunza A, Yanez L, Muruzabal MJ, Gomez-Beltran E andreu R, Leon P, Gomez V, Sanz A, Casasola N, Moreno E, Alegre A, Martin ML, Pedro C, Serrano S, Florensa L and Sole F: Fluorescence in situ hybridization improves the detection of $5 \mathrm{q} 31$ deletion in myelodysplastic syndromes without cytogenetic evidence of $5 \mathrm{q}$. Haematologica 93: 1001-1008, 2008.

21 Streubel B, Sauerland C, Heil G, Freund M, Bartels H, Lengfelder E, Wandt H, Ludwig WD, Nowotny H, Baldus M, Grothaus-Pinke B, Buchner T and Fonatsch C: Correlation of cytogenetic, molecular cytogenetic and clinical findings in 59 patients with ANLL or MDS and abnormalities of the short arm of chromosome 12. Br J Haematol 100: 521-533, 1998.

Received December 23, 2016

Revised January 19, 2017

Accepted January 25, 2017 